CAPITAL SOCIAL Y BIENESTAR SUBJETIVO: UN ESTUDIO DEL ROL DE LA PARTICIPACION EN ORGANIZACIONES SOCIALES EN LA SATISFACCION CON LA VIDAY FELICIDAD EN CIUDADES CHILENAS

\author{
SOCIAL CAPITAL AND SUBJECTIVE WELL-BEING: A STUDY OF \\ THE ROLE OF PARTICIPATION IN SOCIAL ORGANIZATIONS IN LIFE \\ SATISFACTION AND HAPPINESS IN CHILEAN CITIES
}

\title{
JORGE AEDO*
}

Escuela de Administración y Negocios, Universidad de Concepción

\section{ESTEFANY OÑATE*}

Escuela de Administración y Negocios, Universidad de Concepción

\section{MARCELA JAIME**}

Escuela de Administración y Negocios, Universidad de Concepción

\section{CESAR SALAZAR ${ }^{\star \star *}$}

Departamento de Gestión Empresarial, Universidad del Bío-Bío

\begin{abstract}
The concept of welfare has evolved to incorporate subjective elements. One key factor for development is social capital. Participation in organizations promotes a more active social life, with potential positive results on welfare. This work investigates this last association through the estimation of a bivariate ordered probit model by using the World Value Survey for Chile.
\end{abstract}

* Escuela de Administración y Negocios, Universidad de Concepción, Campus Chillán, Chile. E-mail: jorgeaedocurilen@udec.cl; esonate@udec.cl

** Escuela de Administración y Negocios, Universidad de Concepción, Campus Chillán; Núcleo de Investigación en Economía Ambiental (NENRE EfD-Chile) y Center of Applied Ecology and Sustainability (CAPES). Se agradece apoyo financiero a CONICYT PIA/BASAL FB0002. E-mail: mjaime@udec.cl

*** Departamento de Gestión Empresarial, Grupo de Investigación en Análisis Económico Sectorial Aplicado, Grupo de investigación en Centro de Agronegocios, y Centro de Estudios de Nuble, Universidad del Bío-Bío; Núcleo de Investigación en Economía Ambiental (NENRE EfD-Chile), e Interdisciplinary Center for Aquaculture Research (INCAR). Se agradecer apoyo financiero a proyecto FONDAP No 15110027. E-mail: csalazar@ubiobio.cl 
Results show a positive association between participation in organizations and satisfaction with the economic situation, however, in the case of life satisfaction and happiness, this effect is unclear for economic and recreational organizations. This result suggests that subjective well-being do not necessarily respond to the economic situation.

Key words: Subjective well-being, participation, ordered bivariate probit.

JEL Classification: $131, D 71, C 35$.

\section{Resumen}

El concepto de bienestar ha evolucionado a incorporar elementos subjetivos. Un factor clave para el desarrollo es el capital social. La participación en organizaciones promueve una vida social más activa, con efectos positivos en el bienestar. Este trabajo investiga esta asociación, estimando modelos de respuesta ordenada bivariada con datos de la Encuesta Mundial de Valores para Chile. Los resultados muestran una asociación positiva entre participación y satisfacción con la situación económica, sin embargo, para la satisfacción con la vida y felicidad, este efecto no es claro para las organizaciones económicas y recreativas. Este resultado sugiere que bienestar subjetivo no necesariamente responde a la situación económica.

Palabras clave: Bienestar subjetivo, participación, probit bivariado ordenado.

Clasificación JEL: I31, D71, C35.

\section{INTRODUCCION}

Los Gobiernos han promovido por largo tiempo políticas centradas en el crecimiento económico y estabilidad macroeconómica, orientadas principalmente a mejorar el bienestar material de los distintos agentes. Sin embargo, la visión de bienestar de una nación ha evolucionado a una mirada más integral, enmarcada en el concepto de desarrollo humano, donde el bienestar material es una de las dimensiones relevantes, pero no la única (Dolan et al., 2008). El Indice de Desarrollo Humano (IDH) recoge esta mirada integral, reconociendo una serie de capacidades tanto materiales como no materiales que promueven el bienestar de las personas, entre las cuales destacan "gozar de una buena salud", "tener cubiertas las necesidades físicas y materiales básicas" y "tener vínculos significativos con los demás" (IDH, 2012). En particular, esta última capacidad tiene una estrecha relación con la necesidad y la valoración del capital social. El capital social engloba "un conjunto de características de las 
organizaciones sociales tales como interrelaciones, normas y confianza que facilitan la cooperación y coordinación para beneficio mutuo (Putnam, 1995)." Altos niveles de capital social se manifiestan en una participación activa de la sociedad y mayores niveles de confianza, lo cual reduce los costos de transacción, facilita la provisión de bienes públicos y promueve la constitución de actores sociales y sociedades civiles saludables, contribuyendo al desarrollo económico y social de un país (Knack y Keefer, 1997; Kliksberg, 1999; Aker, 2007). Asimismo, sociedades con altos niveles de capital social se caracterizan por presentar mayores niveles de bienestar individual (Herreros, 2002; Puntscher, 2014). Según Veenhoven (1994), el bienestar individual engloba un componente cognitivo, el cual actúa cuando se evalúa la discrepancia percibida entre las aspiraciones y logros, y un componente afectivo, que constituye el agrado experimentado por la persona con sus sentimientos, emociones y estados de ánimo más frecuentes. De esta manera, mayores niveles de bienestar individual subjetivo -o no material- se relacionan con "el estado de las personas que tienen una evaluación positiva de sí mismas (i.e., se sienten satisfechas con sus propias vidas) y de la sociedad en que viven (i.e., se sienten satisfechas con las condiciones que la sociedad les provee para lograr sus objetivos) (IDH, 2012), lo que generalmente se traduce en un mayor nivel de felicidad y satisfacción con la vida (Diener, 1994). Diener y Seligman (2002) y Fleeson et al. (2002) argumentan que las personas más felices se caracterizan por poseer una vida social más activa con una buena red de apoyo social y actividades recreativas en su tiempo libre. Lo anterior sugiere que la participación social es un predictor relevante para explicar el bienestar subjetivo de las personas.

Este trabajo tiene como objetivo analizar el efecto del capital social en el bienestar subjetivo de las personas. Para tal efecto, se utiliza información de la encuesta World Values Survey (WVS) para Chile correspondiente al año 2012, la cual entrega distintas mediciones de bienestar subjetivo basadas en preguntas para determinar el nivel de felicidad y satisfacción de las personas. Este trabajo se centra en la asociatividad, vinculada a la participación activa en organizaciones sociales, como medida de capital social. Para abordar el potencial problema de endogeneidad entre la participación en organizaciones y bienestar subjetivo, se estima un modelo probit ordenado bivariado.

El artículo se estructura como sigue. En la sección 2 se presenta el marco conceptual del bienestar subjetivo y capital social. La sección 3 presenta los datos utilizadas en este estudio. El modelo empírico se detalla en la sección 4. Los resultados se discuten en la sección 5. Finalmente, en la sección 6 se presentan las conclusiones del estudio.

\section{BIENESTAR SUBJETIVO Y CAPITAL SOCIAL}

Los conceptos de calidad de vida, bienestar y felicidad, tienden a utilizarse indistintamente, con mediciones que incorporan componentes objetivos y subjetivos (Diener, 1994). El componente objetivo recae en la propia experiencia de la persona y se relaciona fundamentalmente con la satisfacción con la vida (Diener et al., 2009). De esta manera, la satisfacción con la vida ofrece información sobre las consideraciones 
cognitivas de los hechos y el juicio reflexivo perteneciente al control y la autorealización de los individuos, sobre la base de una comparación entre lo logrado y deseado. Bharadwaj y Wilkening (1977) sugieren que la evaluación entre lo logrado y deseado se realiza de una manera multidimensional en aspectos como la familia, las condiciones de vida, el trabajo, la salud, las actividades de ocio y los ingresos percibidos. Por su parte, el componente subjetivo considera una dimensión global, que incluye una valoración de todos los aspectos vividos y asociados a elementos afectivos, tales como las experiencias agradables y emocionales vividas. Es así como el componente subjetivo se relaciona más estrechamente con el concepto de felicidad, el cual se define como el grado en el cual un individuo evalúa la calidad global de su vida presente como un todo positivamente (Veenhoven, 1994). De esta manera, se entiende que una persona es más feliz cuando en su vida predomina la experiencia afectiva positiva sobre la negativa. En respuesta a lo anterior, la medición del bienestar subjetivo se realiza a través de preguntas sencillas orientadas a recabar información sobre el nivel de felicidad, del estado de salud actual de los individuos, de la satisfacción con la vida, del nivel de autorrealización o de la satisfacción con respecto al ingreso (Calvo y Beytía, 2011).

Por su parte, uno de los activos fundamentales de toda sociedad es su capital social. Existen diversas variaciones al concepto de capital social, pero con aspectos comunes relacionados principalmente con la generación de redes, normas, participación en organizaciones y confianza en las personas e instituciones (Coleman, 1988; Putnam, 1995; Durston, 1999). El capital social actúa como un activo que contribuye a la creación y mantenimiento de una estructura social adecuada que, en colaboración con el capital físico, humano y natural, permite sentar las bases para un desarrollo económico sostenible (Portela y Neira, 2012). Lo anterior considera no solamente aspectos materiales sino también elementos asociados al bienestar subjetivo de las personas (Herreros, 2002; Puntscher, 2014).

Pese a la importancia de ambos conceptos, el análisis de la relación existente entre el capital social y bienestar subjetivo es relativamente reciente. Helliwell (2001) encuentra una relación positiva entre el capital social y una serie de variables no económicas, variables que potencialmente aumentan el nivel y la calidad de vida de la población. Asimismo, Hudson (2006) utiliza la encuesta Eurobarómetro para explorar la confianza en instituciones como medida de capital social, encontrando una relación positiva entre el nivel de confianza institucional y la satisfacción de las personas. Portela y Neira (2012) investigan esta relación para el caso de España. Los autores utilizan la Encuesta Social Europea (ESS) para medir el capital social con base en preguntas para determinar la magnitud de las redes sociales de contacto y el nivel de confianza. Los resultados señalan que estas medidas son determinantes fundamentales del bienestar, medido por medio del nivel de felicidad, satisfacción y bienestar general. En un trabajo similar, Puntscher (2014) explora los determinantes del bienestar subjetivo con base en indicadores de felicidad y satisfacción con la vida para Europa, haciendo énfasis en los niveles generales de confianza, asociatividad, confianza en las instituciones y los vínculos sociales históricos creados en Europa. 
Los autores estiman un modelo de error espacial para considerar la incidencia de las regiones vecinas en el bienestar. Los autores plantean que los altos niveles de satisfacción con la vida y felicidad observados en los países Nórdicos pueden atribuirse a la alta dotación de capital social, encontrando un impacto positivo y significativo entre las distintas medidas de capital social y de bienestar subjetivo, particularmente para la dimensión de felicidad.

Para el caso chileno, la evidencia es escasa, con la excepción de Ponce et al. (2014), quienes exploran la asociación entre el capital social medido como la participación en organizaciones y la percepción de satisfacción personal de los adultos mayores. Los autores encuentran que el bienestar subjetivo no depende exclusivamente de factores objetivos como el ingreso y la salud, sino también del grado de participación en la vida social. Existen también otros estudios para el caso Chileno enfocados en explorar los efectos del capital social en el bienestar material de las personas. Al respecto, Salazar y Jaime (2009) investigan la incidencia del capital social, medido como participación en organizaciones, en el bienestar material de las personas en Chile. Los resultados muestran un efecto positivo y estadísticamente significativo de la participación en organizaciones económicas en el bienestar material de las personas, mientras que la participación en organizaciones recreativas y vecinales no tiene incidencia en esta medida. Los resultados de este trabajo evidencian también la presencia de endogeneidad entre las organizaciones recreativas y las medidas de bienestar material, sugiriendo características de bien superior para la participación en este tipo de organizaciones en Chile.

\section{ESTRATEGIA EMPIRICA}

En esta sección, se presenta el modelo empírico para explorar el efecto del capital social en el bienestar subjetivo de las personas. Debido a la potencial endogeneidad del capital social en un modelo explicando el bienestar subjetivo, los resultados obtenidos al utilizar el estimador de Mínimos Cuadrados Ordinarios (MCO) serán sesgados e inconsistentes. Este problema puede ser resuelto al utilizar métodos de estimación de ecuaciones simultáneas. Dada la naturaleza de la variable dependiente - la cual es medida en forma categórica y ordenada-, y dada la estructura dicotómica de la variable participación (proxy del capital social), se propone la estimación de un modelo probit ordenado bivariado. Este modelo es parte de una familia de modelos multiecuacionales, que considera un sistema de ecuaciones determinado por procesos subyacentes comunes no observados de la siguiente forma:

$$
\begin{gathered}
y_{1}^{*}=x_{2}^{*} \beta+z_{1} \gamma+\varepsilon_{1} \\
x_{2}^{*}=z_{2} \gamma+\varepsilon_{2}
\end{gathered}
$$


Donde $y_{1}^{*}$ y $x_{2}^{*}$ son variables latentes para el bienestar subjetivo y participación, respectivamente. Pese a que las variables latentes no son observadas, es posible identificar las elecciones de los individuos sobre los diferentes niveles de bienestar subjetivo y de participación en organizaciones, de acuerdo a la siguiente regla:

$$
\begin{gathered}
y_{1}=1 \text { si } y_{1}^{*} \leq \propto_{1} \\
y_{1}=2 \text { si } \propto_{1} \leq y_{1}^{*}<\propto_{2} \\
y_{1}=3 \text { si } \propto_{2} \leq y_{1}^{*}<\propto_{3} \\
(\ldots) \\
y_{1}=J \text { si } \propto_{j} \leq y_{1}^{*} \\
x_{2}=1 \text { si } x_{2}^{*}>0 \\
x_{2}=0 \text { si } x_{2}^{*} \leq 0
\end{gathered}
$$

Específicamente, $y_{1}$ corresponde a una variable ordenada que define el nivel de bienestar subjetivo asociado a un individuo y $x_{2}$ es una variable dummy que denota la participación de un individuo en una organización de distinta índole; $z_{1}$ y $z_{2}$ denotan vectores de variables explicativas caracterizando las decisiones de los individuos con respecto a los niveles de bienestar subjetivo y participación en organizaciones, respectivamente. No existen restricciones en relación a las variables contenidas en $z_{1}$ y $z_{2}$. La característica más importante de este modelo es la relación entre los errores. En particular, si los términos error en las ecuaciones son independientes uno del otro, se tiene que $\operatorname{Cov}\left[\varepsilon_{1}, \varepsilon_{2}\right]=0$, y el modelo puede ser estimado por medio de dos regresiones separadas. Lo anterior entregaría evidencia de que las decisiones de los individuos son independientes. Por el contrario, si los errores se encuentran relacionados, se tiene que $\operatorname{Cov}\left[\varepsilon_{1}, \varepsilon_{2}\right] \neq 0$, por lo que las decisiones de los individuos estarían influenciadas por un proceso subyacente similar. Con el objeto de considerar esta relación entre las ecuaciones, los términos error pueden ser representados como sigue:

$$
\begin{aligned}
\varepsilon_{1 i} & =\eta_{i}+u_{1 i} \\
\varepsilon_{2 i} & =\eta_{i}+u_{2 i}
\end{aligned}
$$

Como puede ser visto a partir de esta expresión el término error está conformado por un componente que es único a cada ecuación (denotado por $u_{j i} ; j=1,2$ ), y otro que es común a ambos (denotado por $\eta_{i}$ ). Este último componente nos permite capturar la relación entre las ecuaciones (Greene, 1999). Si se asume que los términos error se 
distribuyen normal, entonces $\varepsilon_{1}$ y $\varepsilon_{2}$ no solo son normales sino también dependientes. Asumamos también que $\rho$ denota el grado de correlación existente entre ambos términos de error. Debido a que estamos interesados en la probabilidad conjunta de $y_{1}$ y $x_{2}$, y considerando que los términos error se encuentran normalmente distribuidos, las ecuaciones (2)-(3) pueden se estimadas consistentemente a través de un modelo probit ordenado bivariado. La significancia estadística del coeficiente $\rho$ proveerá evidencia de una interrelación entre las decisiones de los individuos; es decir, nos informará que el bienestar subjetivo y la decisión de participar en organizaciones están determinadas por el mismo proceso subyacente. Por su parte, si $\rho$ no es estadísticamente distinto de cero, se tendrá evidencia de que el proceso subyacente que explica el bienestar subjetivo es distinto al que explica la participación, en cuyo caso ambos procesos se encontrarán caracterizados por ecuaciones diferentes.

\section{DATOS}

Este estudio utiliza información, a nivel individual, proveniente de la encuesta mundial de valores (World Value Survey, 2012). Este instrumento consiste en una encuesta que es aplicada en forma regular en diferentes países del mundo, y que busca medir las experiencias de los ciudadanos, así como sus actitudes, evaluaciones y preferencias sobre aspectos valóricos, de gobierno, política, etc. Entre las preguntas destacan aspectos relacionados con el apoyo a la democracia, la tolerancia hacia los extranjeros y minorías étnicas, el apoyo a la igualdad de género, el papel de la religión y los cambios en niveles de religiosidad, el impacto de la globalización, las actitudes hacia el medio ambiente, el trabajo, la familia, la política, la identidad nacional, la cultura, la diversidad, la inseguridad y el bienestar del individuo.

En este estudio, en particular, se utiliza la encuesta realizada en Chile entre los años 2011-2012, que fue aplicada a una muestra de 1.000 habitantes, mayores de 18 años, y residentes en 30 ciudades a lo largo del territorio nacional. En particular, esta encuesta entrega información respecto a las percepciones de los individuos con relación a diferentes medidas de bienestar subjetivo (i.e., felicidad, satisfacción con la vida y satisfacción económica). Las preguntas específicas son las siguientes: a) En general, usted diría que es... Medido en una escala de uno (muy feliz) a cuatro (nada feliz); b) Considerando todas las cosas, ¿Cuán satisfecho está usted con su vida en este momento? Medido en una escala de uno (completamente insatisfecho) a diez (completamente satisfecho); c) ¿Qué tan satisfecho o insatisfecho está usted con la situación económica en su hogar? Medido en una escala de uno (completamente insatisfecho) a diez (completamente satisfecho). Las respuestas se ordenan de manera categórica. La Tabla 1 muestra los porcentajes de respuestas para cada nivel y medida de bienestar subjetivo. 
TABLA 1

MEDIDAS DE BIENESTAR SUBJETIVO

\begin{tabular}{|c|c|}
\hline Variables & $\begin{array}{c}\text { Porcentaje } \\
(\%)\end{array}$ \\
\hline \multicolumn{2}{|l|}{ Felicidad } \\
\hline Muy feliz & 24,47 \\
\hline Bastante Feliz & 60,28 \\
\hline Poco Feliz & 14,44 \\
\hline Nada Feliz & 0,80 \\
\hline \multicolumn{2}{|l|}{ Satisfacción con la vida } \\
\hline Completamente Satisfecho $=10$ & 11,23 \\
\hline 9 & 12,45 \\
\hline 8 & 26,42 \\
\hline 7 & 21,26 \\
\hline 6 & 12,75 \\
\hline 5 & 8,10 \\
\hline 4 & 4,15 \\
\hline 3 & 2,1 \\
\hline 2 & 0,91 \\
\hline Completamente insatisfecho $=1$ & 0,61 \\
\hline \multicolumn{2}{|l|}{ Satisfacción económica } \\
\hline Completamente Satisfecho $=10$ & 5,02 \\
\hline 9 & 5,52 \\
\hline 8 & 14,14 \\
\hline 7 & 16,65 \\
\hline 6 & 15,25 \\
\hline 5 & 21,46 \\
\hline 4 & 11,74 \\
\hline 3 & 6,32 \\
\hline 2 & 1,71 \\
\hline Completamente insatisfecho $=1$ & 2,21 \\
\hline
\end{tabular}

Fuente: Elaboración propia.

Para identificar una medida de participación en organizaciones - proxy del capital social-, se utiliza una pregunta única que recaba información sobre si el individuo es miembro o no de una serie de organizaciones listadas como alternativas de respuestas: iglesia u organización religiosa, organización deportiva/recreativa; organización educativa, artística, musical, cultural; sindicato; partido político; organización ecologista; asociaciones profesionales; organización humanitaria o de caridad; organización de consumidores; grupo de autoayuda ayuda mutua; otras organizaciones. Siguiendo PNUD (2000) y Salazar y Jaime (2009), se realiza una clasificación de las organizaciones 
en tres categorías: organizaciones con fines económicos; en las que los individuos persiguen mejoras en sus condiciones laborales, productivas, tecnológicas, capacidad de negociación y representación política; organizaciones de auto ayuda, en las que los individuos buscan un mejoramiento en las condiciones físicas (acceso a servicios básicos y equipamiento comunitario) y del medio ambiente social (seguridad y relación con los vecinos) de sus comunidades de residencia; y organizaciones recreativo-cultural, que busca la creación de instancias para el uso del tiempo libre y el desarrollo de formas de expresión artística y cultural.

Adicionalmente, se incluyen una serie de variables de control. En primer lugar se incorporan un conjunto de características individuales y de localización de los encuestados. De esta manera se considera la variable Sexo como una variable dummy que toma el valor de 1 si el encuestado es hombre y 0 si es mujer; Edad como los años de vida del encuestado; Educa como el nivel educacional más alto alcanzado, tomando valores desde 0 , sin educación, hasta 9 , con educación universitaria completa; Edad_edu se define como la edad de término de educación de tiempo completo; Estado Civil es una variable dummy que identifica con el valor de 1 si la persona es casada y 0 en otro caso; $N^{o}$ niños como la cantidad de hijos del encuestado; y Zona norte, Zona Centro, Zona Sur son variables dummies por zona, quedando como base la zona Metropolitana.

En relación con las variables que capturan diferencias en la situación económica de los encuestados, se incorpora Ocupado siendo una variable dicotómica que toma el valor de 1 si la persona se encuentra ocupada y 0 en otro caso; Ahorro, que mide la capacidad de ahorro del individuo como una variable dummy tomando el valor de 1 si el encuestado manifiesta haber ahorrado y 0 en otro caso; Ingresos, que recoge la capacidad adquisitiva del encuestado a través de su percepción en relación al decil en que se encuentra su hogar, donde 1 indica el "decil más bajo de ingresos" y 10 el "decil más alto de ingresos" de su país

Para analizar las percepciones valóricas y creencias de los individuos, se incluyen las variables Religioso, que hace referencia a si la persona profesa alguna religión, tomando el valor de 1 si es miembro y 0 en caso contrario; y Patriotismo, variable que mide cuán orgulloso se siente el encuestado de ser chileno en una escala de 1 a 5.

Se definen dos variables instrumentales, las cuales se espera que afecten la participación, pero no el bienestar subjetivo. Estos son: Seguridad, que se relaciona con la percepción de la seguridad del barrio, tomando valores en una escala de 1 a 4 , donde 1 es muy seguro y 4 muy inseguro; y Robo, que se relaciona con la percepción de la frecuencia con que ocurren robos en el barrio, tomando valores en una escala de 1 a 4 , donde 1 es no muy frecuente y 4 muy frecuente.

La elección de ambas variables como instrumentos se encuentra respaldada en la literatura empírica, la cual sugiere que la participación en organizaciones puede ser afectada por la confianza (Glaeser et al., 2002; Lederman, 2005), y a que la confianza permite explicar otros resultados económicos, incluyendo la formación de grupos (Haddad y Maluccio, 2003). De acuerdo con esta literatura, una percepción 
negativa respecto a la seguridad en el barrio afectaría negativamente la confianza en las personas, por lo que los individuos podrían sentirse desincentivados a generar relaciones personales en grupos y organizaciones. Alternativamente, una disminución en la confianza podría generar una menor participación en organizaciones que tengan por objetivo la búsqueda de objetivos comunes para sus miembros. Siguiendo a Diener et al., (2009) las medidas de bienestar subjetivo (i.e., satisfacción con la vida, satisfacción económica) “...incluyen consideraciones cognitivas de los hechos y el juicio reflexivo perteneciente al control y la autorrealización de los individuos, sobre la base de una comparación entre lo logrado y deseado", por lo que los instrumentos propuestos permitirían explicar el bienestar subjetivo a través de la participación en organizaciones, y no de forma directa. Asimismo, debido a que el componente subjetivo de las medidas de bienestar “....considera una dimensión global, que incluye una valoración de todos los aspectos vividos y asociados a elementos afectivos, tales como las experiencias agradables y emocionales vividas", requiere de una evaluación de múltiples dimensiones, por lo que es muy probable que los instrumentos propuestos satisfagan también la restricción de exclusión en el caso de la felicidad. Existe evidencia de la utilización de instrumentos similares en otros estudios (e.g., Salazar y Jaime, 2009). La Tabla 2 presenta las estadísticas descriptivas para las variables de participación y controles.

\section{TABLA 2}

ESTADISTICA DESCRIPTIVA DE LAS VARIABLES EXPLICATIVAS

\begin{tabular}{|l|c|c|c|c|}
\hline \multicolumn{1}{|c|}{ Variables } & Media & $\begin{array}{c}\text { Desviación } \\
\text { Estándar }\end{array}$ & Mínimo & Máximo \\
\hline Participación en organizaciones & & & & \\
$\quad$ Todas & 0,58 & 0,49 & 0 & 1 \\
Económicas & 0,17 & 0,38 & 0 & 1 \\
Recreativas & 0,32 & 0,46 & 0 & 1 \\
De autoayuda & 0,41 & 0,49 & 0 & 1 \\
& & & & \\
Variables de control & & & & \\
Sexo & 0,49 & 0,50 & 0 & 1 \\
Edad & 43,89 & 1,62 & 18 & 85 \\
Educa & 5,48 & 2,04 & 1 & 9 \\
Edad_educa & 19,24 & 4,46 & 7 & 48 \\
Casado & 0,47 & 0,49 & 0 & 1 \\
N ${ }^{\circ}$ niños & 1,91 & 1,54 & 0 & 8 \\
Ocupado & 0,601 & 0,48 & 0 & 1 \\
Ahorro & 0,82 & 0,37 & 0 & 1 \\
Ingresos & 4,80 & 1,72 & 1 & 10 \\
Religioso & 0,76 & 0,42 & 0 & 1 \\
Patriotismo & 1,58 & 0,66 & 1 & 5 \\
\hline
\end{tabular}




\begin{tabular}{|l|c|c|c|c|}
\hline \multicolumn{1}{|c|}{ Variables } & Media & $\begin{array}{c}\text { Desviación } \\
\text { Estándar }\end{array}$ & Mínimo & Máximo \\
\hline Seguridad & 2,34 & 0,73 & 1 & 4 \\
Robo & 2,80 & 0,84 & 1 & 4 \\
Zona Norte & 0,13 & 0,34 & 0 & 1 \\
Zona Centro & 0,16 & 0,37 & 0 & 1 \\
Zona Sur & 0,18 & 0,38 & 0 & 1 \\
Zona Metropolitana & 0,51 & 0,49 & 0 & 1 \\
\hline $\mathrm{N}^{\circ}$ observaciones &
\end{tabular}

Fuente: Elaboración propia.

\section{RESULTADOS}

En primer lugar, se discuten los resultados de las variables utilizadas para instrumentar la participación. Los resultados muestran el signo esperado. En particular, una percepción más negativa sobre la seguridad del barrio y sobre la frecuencia de robos en el entorno disminuye la probabilidad de ser miembro de una organización social, principalmente de auto ayuda y recreativa. Lo anterior podría deberse a que los individuos que perciben que habitan entornos más seguros están más dispuestos a confiar en los demás y se sienten más en capacidad de explorar y concretar relaciones sociales, las cuales generalmente se canalizan en organizaciones sociales donde participan personas también de su entorno. La Tabla 3 presenta las estimaciones para el bienestar subjetivo medido como felicidad para los distintos tipos de organizaciones.

TABLA 3

ESTIMACIONES PARA EL BIENESTAR SUBJETIVO MEDIDO COMO FELICIDAD

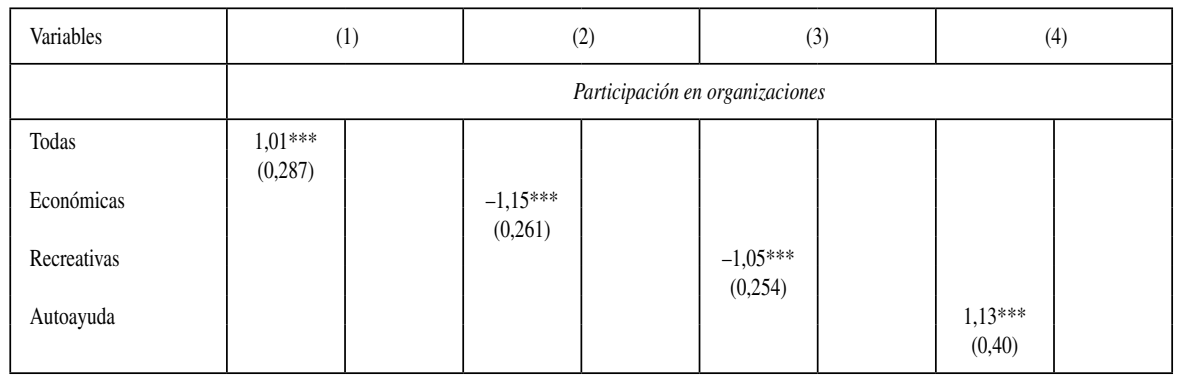




\begin{tabular}{|c|c|c|c|c|c|c|c|c|}
\hline \multirow[t]{2}{*}{ Variables } & \multicolumn{2}{|c|}{ (1) } & \multicolumn{2}{|c|}{ (2) } & \multicolumn{2}{|c|}{ (3) } & \multicolumn{2}{|c|}{ (4) } \\
\hline & \multicolumn{8}{|c|}{ Variables de control } \\
\hline \multirow[t]{2}{*}{ Hombre } & 0,038 & $0,27 * *$ & $0,18^{* *}$ & $0,19^{*}$ & $0,31 * * *$ & $0,55^{* * *}$ & $0,20^{*}$ & $-0,19 *$ \\
\hline & $(0,095)$ & $(0,102)$ & $(0,0918)$ & $(0,11)$ & $(0,0993)$ & $(0,110)$ & $(0,08)$ & $(0,103)$ \\
\hline \multirow[t]{2}{*}{ Edad } & $-0,0142$ & $-0,0152$ & $-0,0141$ & 0,021 & $-0,0371 * *$ & $-0,05^{* * *}$ & $-0,026$ & 0,0174 \\
\hline & $(0,016)$ & $(0,019)$ & $(0,018)$ & $(0,02)$ & $(0,018)$ & $(0,021)$ & $(0,02)$ & $(0,020)$ \\
\hline \multirow[t]{2}{*}{ Edad2 } & $5,41 \mathrm{e} 05$ & 0,0001 & $7,14 \mathrm{e} 05$ & $-0,000$ & 0,000252 & $0,0005^{* *}$ & 0,000 & $-0,0001$ \\
\hline & $(0,001)$ & $(0,0002)$ & $(0,0001)$ & $(0,0002)$ & $(0,0002)$ & $(0,0002)$ & $(0,0001)$ & $(0,0002)$ \\
\hline \multirow[t]{2}{*}{ Educa } & 0,0133 & 0,0587 & 0,0343 & 0,0273 & 0,0402 & 0,0373 & $-0,00112$ & $0,0783^{*}$ \\
\hline & $(0,0362)$ & $(0,0453)$ & $(0,0349)$ & $(0,0445)$ & $(0,0375)$ & $(0,0476)$ & $(0,0394)$ & $(0,0406)$ \\
\hline \multirow[t]{2}{*}{ Edad educa } & 0,00533 & 0,0120 & 0,0244 & $0,0467 * *$ & 0,0179 & 0,0244 & 0,0133 & $-0,00803$ \\
\hline & $(0,0161)$ & $(0,0211)$ & $(0,0159)$ & $(0,0185)$ & $(0,0183)$ & $(0,0219)$ & $(0,0155)$ & $(0,0172)$ \\
\hline \multirow[t]{2}{*}{ Casado } & 0,127 & $-0,108$ & 0,0955 & $-0,00523$ & 0,0961 & 0,0322 & 0,101 & $-0,00959$ \\
\hline & $(0,0924)$ & $(0,107)$ & $(0,0930)$ & $(0,120)$ & $(0,0931)$ & $(0,116)$ & $(0,0926)$ & $(0,107)$ \\
\hline \multirow[t]{2}{*}{$\mathrm{N}^{0}$ niños } & $-0,0332$ & 0,0667 & 0,00343 & 0,0316 & 0,000735 & 0,0435 & $-0,0391$ & 0,0659 \\
\hline & $(0,0380)$ & $(0,0416)$ & $(0,0383)$ & $(0,0499)$ & $(0,0365)$ & $(0,0468)$ & $(0,0396)$ & $(0,0412)$ \\
\hline \multirow[t]{2}{*}{ Ocupado } & 0,109 & $-0,43 * * *$ & 0,0226 & 0,202 & $-0,0926$ & $-0,142$ & 0,137 & $-0,42 * * *$ \\
\hline & $(0,111)$ & $(0,116)$ & $(0,105)$ & $(0,140)$ & $(0,102)$ & $(0,121)$ & $(0,125)$ & $(0,121)$ \\
\hline \multirow[t]{2}{*}{ Ahorro } & $0,314 * * *$ & $-0,262^{*}$ & $0,300^{* *}$ & 0,267 & 0,161 & $-0,198$ & $0,310 * * *$ & $-0,236^{*}$ \\
\hline & $(0,110)$ & $(0,136)$ & $(0,118)$ & $(0,168)$ & $(0,122)$ & $(0,144)$ & $(0,113)$ & $(0,134)$ \\
\hline \multirow[t]{2}{*}{ Ingresos } & 0,0326 & 0,0453 & $0,0611 * *$ & 0,0304 & $0,0570^{* *}$ & 0,0184 & 0,0259 & $0,0672 * *$ \\
\hline & $(0,0289)$ & $(0,0313)$ & $(0,0287)$ & $(0,0358)$ & $(0,0284)$ & $(0,0347)$ & $(0,0297)$ & $(0,0333)$ \\
\hline \multirow[t]{2}{*}{ Religioso } & $-0,101$ & $0,501 * * *$ & 0,0618 & $-0,0175$ & 0,107 & 0,117 & $-0,162$ & $0,619^{* * * *}$ \\
\hline & $(0,117)$ & $(0,118)$ & $(0,0984)$ & $(0,129)$ & $(0,0958)$ & $(0,122)$ & $(0,140)$ & $(0,123)$ \\
\hline \multirow[t]{2}{*}{ Patriotismo } & $-0,23 * * *$ & 0,0454 & $-0,21$ *** & 0,00962 & $-0,204 * * *$ & 0,0125 & $-0,20^{* * *}$ & $-0,0388$ \\
\hline & $(0,0614)$ & $(0,0709)$ & $(0,0682)$ & $(0,0864)$ & $(0,0707)$ & $(0,0819)$ & $(0,0703)$ & $(0,0755)$ \\
\hline \multirow[t]{2}{*}{ Seguridad } & & $-0,178^{* *}$ & & 0,0484 & & $-0,0824$ & & $-0,176 * *$ \\
\hline & & $(0,0725)$ & & $(0,0895)$ & & $(0,0896)$ & & $(0,0753)$ \\
\hline \multirow[t]{2}{*}{ Robo } & & $-0,0117$ & & $-0,0639$ & & $-0,114$ & & 0,0298 \\
\hline & & $(0,0650)$ & & $(0,0780)$ & & $(0,0708)$ & & $(0,0692)$ \\
\hline \multirow[t]{2}{*}{ Zona Norte } & $-0,263^{* *}$ & $0,623^{* * * *}$ & 0,0583 & $0,382 * *$ & 0,191 & $0,774 * * *$ & $-0,199$ & $0,424 * * *$ \\
\hline & $(0,128)$ & $(0,141)$ & $(0,123)$ & $(0,168)$ & $(0,136)$ & $(0,151)$ & $(0,129)$ & $(0,142)$ \\
\hline Zona Centro & $-0,0506$ & $0,450^{* * *}$ & 0,175 & $0,272 *$ & $0,339 * * *$ & $0,754 * * *$ & $-0,0184$ & $0,319^{* *}$ \\
\hline & $(0,130)$ & $(0,130)$ & $(0,111)$ & $(0,147)$ & $(0,120)$ & $(0,135)$ & $(0,136)$ & $(0,136)$ \\
\hline Zona Sur & $-0,183$ & $0,871^{* * *}$ & $0,288^{* *}$ & $0,601 * * *$ & $0,484 * * *$ & $1,097^{* * *}$ & $-0,192$ & $0,738^{* * *}$ \\
\hline & $(0,139)$ & $(0,137)$ & $(0,116)$ & $(0,146)$ & $(0,139)$ & $(0,136)$ & $(0,166)$ & $(0,130)$ \\
\hline Cut11 & $-2,12 * * *$ & & $-1,95 * * *$ & & $-2,74 * * *$ & & $-2,42 * * *$ & \\
\hline & $(0,480)$ & & $(0,487)$ & & $(0,475)$ & & $(0,500)$ & \\
\hline Cut12 & $-0,780^{*}$ & & $-0,517$ & & $-1,34 * * *$ & & $-1,078 * *$ & \\
\hline & $(0,438)$ & & $(0,461)$ & & $(0,460)$ & & $(0,433)$ & \\
\hline Cut13 & $0,892 * *$ & & $1,164 * * *$ & & 0,277 & & 0,597 & \\
\hline & $(0,423)$ & & $(0,440)$ & & $(0,480)$ & & $(0,430)$ & \\
\hline Cut21 & 0,222 & & $3,48 * * *$ & & $-0,029$ & & $1,227 * *$ & \\
\hline & $(0,610)$ & & $(0,660)$ & & $(0,655)$ & & $(0,606)$ & \\
\hline Athrho & $\begin{array}{c}-0,65^{* * *} \\
(0,253)\end{array}$ & & $\begin{array}{c}0,760 * * * \\
(0,227)\end{array}$ & & $\begin{array}{c}0,816^{* * *} \\
(0,263)\end{array}$ & & $\begin{array}{c}-0,661^{*} \\
(0,384)\end{array}$ & \\
\hline Log Pseudolikelihood & $-1200,52$ & & $-1064,55$ & & $-1144,0$ & & $-1192,63$ & \\
\hline Wald chi2 & 111,82 & & 119,52 & & 125,33 & & 114,20 & \\
\hline Obs. & 793 & & 793 & & 793 & & 793 & \\
\hline
\end{tabular}

Nota: Errores estándar robustos entre paréntesis. $* * * \mathrm{p}<0,01, * * \mathrm{p}<0,05,{ }^{*} \mathrm{p}<0,1$. 
En relación a las variables de control, los resultados muestran que los individuos que declaran realizar esfuerzos para ahorrar reportan mayores niveles de felicidad. Más ahorro permite mantener un consumo estable a pesar de sufrir eventos inesperados que reducen la capacidad de generación de ingresos, reduciendo de este modo la incertidumbre asociada a sufrir en un futuro experiencias financieras negativas. Asimismo, los resultados muestran que los individuos que manifiestan sentirse más orgullosos de su nacionalidad reportan niveles de felicidad más altos. Lo anterior podría estar relacionado con un tema de identidad, pero también con un mayor optimismo por parte de estos individuos en relación con los problemas del país.

En relación con las variables de participación, se constata una asociación positiva entre la participación en organizaciones en general, y de autoayuda, en particular, con los niveles de felicidad. Sin embargo, las estimaciones sugieren una relación negativa y significativa entre la participación en organizaciones económicas y recreativas con los niveles de felicidad. La Tabla 4 muestra las estimaciones para el bienestar subjetivo medido como satisfacción con la vida para los distintos tipos de organizaciones.

\section{TABLA 4}

ESTIMACIONES PARA EL BIENESTAR SUBJETIVO MEDIDO

COMO SATISFACCION CON LA VIDA

\begin{tabular}{|c|c|c|c|c|c|c|c|c|}
\hline \multirow[t]{2}{*}{ Variables } & \multicolumn{2}{|c|}{ (1) } & \multicolumn{2}{|c|}{ (2) } & \multicolumn{2}{|c|}{ (3) } & \multicolumn{2}{|c|}{ (4) } \\
\hline & \multicolumn{8}{|c|}{ Participación en organizaciones } \\
\hline $\begin{array}{l}\text { Todas } \\
\text { Económicas } \\
\text { Recreativas } \\
\text { Autoayuda }\end{array}$ & $\begin{array}{c}1,263^{* * * *} \\
(0,154)\end{array}$ & & $\begin{array}{l}-0,638 \\
(0,713)\end{array}$ & & $\begin{array}{c}-1,18 * * * \\
(0,149)\end{array}$ & & $\begin{array}{c}1,383^{* * *} \\
(0,108)\end{array}$ & \\
\hline Autoayuda & \multicolumn{8}{|c|}{ Variables de control } \\
\hline Hombre & $\begin{array}{c}0,0241 \\
(0,0806)\end{array}$ & $\begin{array}{c}0,217^{* *} \\
(0,101)\end{array}$ & $\begin{array}{l}0,202^{* *} \\
(0,0826)\end{array}$ & $\begin{array}{l}0,203^{*} \\
(0,121)\end{array}$ & $\begin{array}{r}0,355 * * * \\
(0,0830)\end{array}$ & $\begin{array}{c}0,572 * * * \\
(0,110)\end{array}$ & $\begin{array}{c}0,231 \text { *** } \\
(0,0791)\end{array}$ & $\begin{array}{r}-0,206 * * \\
(0,0997)\end{array}$ \\
\hline Edad & $\begin{array}{l}0,00682 \\
(0,0151)\end{array}$ & $\begin{array}{c}-0,00954 \\
(0,0189)\end{array}$ & $\begin{array}{l}0,00555 \\
(0,0174)\end{array}$ & $\begin{array}{c}0,0138 \\
(0,0244)\end{array}$ & $\begin{array}{l}-0,0199 \\
(0,0175)\end{array}$ & $\begin{array}{c}-0,0584 * * * \\
(0,0214)\end{array}$ & $\begin{array}{c}-0,00844 \\
(0,0146)\end{array}$ & $\begin{array}{c}0,0147 \\
(0,0188)\end{array}$ \\
\hline Edad2 & $\begin{array}{c}-6,44 \mathrm{e}-05 \\
(0,0001)\end{array}$ & $\begin{array}{r}8,47 e-05 \\
(0,0001)\end{array}$ & $\begin{array}{c}-4,40 \mathrm{e}-05 \\
(0,0001)\end{array}$ & $\begin{array}{c}-5,85 e-05 \\
(0,0002)\end{array}$ & $\begin{array}{c}0,000162 \\
(0,0001)\end{array}$ & $\begin{array}{c}0,000491 * * \\
(0,0002)\end{array}$ & $\begin{array}{l}5,89 \mathrm{e} 05 \\
(0,0001)\end{array}$ & $\begin{array}{l}-9,96 \mathrm{e} 05 \\
(0,0001)\end{array}$ \\
\hline Educa & $\begin{array}{l}-0,0285 \\
(0,0311)\end{array}$ & $\begin{array}{c}0,0508 \\
(0,0414)\end{array}$ & $\begin{array}{c}-0,00505 \\
(0,0295)\end{array}$ & $\begin{array}{c}0,0197 \\
(0,0481)\end{array}$ & $\begin{array}{l}0,00482 \\
(0,0333)\end{array}$ & $\begin{array}{c}0,0468 \\
(0,0468)\end{array}$ & $\begin{array}{l}-0,0456 \\
(0,0305)\end{array}$ & $\begin{array}{c}0,0610 \\
(0,0388)\end{array}$ \\
\hline Edad educa & $\begin{array}{c}0,0138 \\
(0,0139)\end{array}$ & $\begin{array}{c}0,0150 \\
(0,0188)\end{array}$ & $\begin{array}{r}0,0327 * * \\
(0,0154)\end{array}$ & $\begin{array}{c}0,0469^{* *} \\
(0,0189)\end{array}$ & $\begin{array}{l}0,0310^{*} \\
(0,0158)\end{array}$ & $\begin{array}{c}0,0206 \\
(0,0215)\end{array}$ & $\begin{array}{l}0,0230^{*} \\
(0,0130)\end{array}$ & $\begin{array}{l}0,00341 \\
(0,0162)\end{array}$ \\
\hline Casado & $\begin{array}{l}0,00444 \\
(0,0827)\end{array}$ & $\begin{array}{l}-0,0635 \\
(0,104)\end{array}$ & $\begin{array}{l}-0,0387 \\
(0,0848)\end{array}$ & $\begin{array}{c}-0,0255 \\
(0,121)\end{array}$ & $\begin{array}{l}-0,0280 \\
(0,0834)\end{array}$ & $\begin{array}{l}0,0264 \\
(0,111)\end{array}$ & $\begin{array}{l}-0,0250 \\
(0,0807)\end{array}$ & $\begin{array}{l}0,00596 \\
(0,103)\end{array}$ \\
\hline
\end{tabular}




\begin{tabular}{|c|c|c|c|c|c|c|c|c|}
\hline Variables & & ) & & & & & & \\
\hline $\mathrm{N}^{0}$ niños & $\begin{array}{c}-0,062 * * \\
(0,0306)\end{array}$ & $\begin{array}{c}0,0553 \\
(0,0369)\end{array}$ & $\begin{array}{l}-0,0302 \\
(0,0350)\end{array}$ & $\begin{array}{c}0,0539 \\
(0,0514)\end{array}$ & $\begin{array}{l}-0,0188 \\
(0,0323)\end{array}$ & $\begin{array}{c}0,0325 \\
(0,0436)\end{array}$ & $\begin{array}{c}-0,069 * * \\
(0,0302)\end{array}$ & $\begin{array}{l}0,0636^{*} \\
(0,0361)\end{array}$ \\
\hline Ocupado & $\begin{array}{c}0,157^{*} \\
(0,0944)\end{array}$ & $\begin{array}{c}-0,402 * * * \\
(0,112)\end{array}$ & $\begin{array}{c}-0,00983 \\
(0,108)\end{array}$ & $\begin{array}{l}0,248^{*} \\
(0,149)\end{array}$ & $\begin{array}{l}-0,0935 \\
(0,0943)\end{array}$ & $\begin{array}{l}-0,120 \\
(0,123)\end{array}$ & $\begin{array}{l}0,187 * * \\
(0,0930)\end{array}$ & $\begin{array}{c}-0,45^{* * *} \\
(0,113)\end{array}$ \\
\hline Ahorro & $\begin{array}{c}0,289 * * * \\
(0,102)\end{array}$ & $\begin{array}{c}-0,227 * \\
(0,137)\end{array}$ & $\begin{array}{l}0,261 * * \\
(0,104)\end{array}$ & $\begin{array}{c}0,227 \\
(0,176)\end{array}$ & $\begin{array}{c}0,124 \\
(0,103)\end{array}$ & $\begin{array}{c}-0,247^{*} \\
(0,140)\end{array}$ & $\begin{array}{c}0,280^{* * *} \\
(0,103)\end{array}$ & $\begin{array}{l}-0,210 \\
(0,134)\end{array}$ \\
\hline Ingresos & $\begin{array}{c}0,0183 \\
(0,0263)\end{array}$ & $\begin{array}{c}0,0395 \\
(0,0313)\end{array}$ & $\begin{array}{l}0,0490^{*} \\
(0,0270)\end{array}$ & $\begin{array}{c}0,0478 \\
(0,0445)\end{array}$ & $\begin{array}{l}0,0475^{*} \\
(0,0265)\end{array}$ & $\begin{array}{c}0,0217 \\
(0,0329)\end{array}$ & $\begin{array}{l}0,00863 \\
(0,0266)\end{array}$ & $\begin{array}{c}0,0606^{*} \\
(0,0335)\end{array}$ \\
\hline Religioso & $\begin{array}{c}-0,211^{* *} \\
(0,0904)\end{array}$ & $\begin{array}{c}0,464 * * * \\
(0,113)\end{array}$ & $\begin{array}{c}-0,00999 \\
(0,0901)\end{array}$ & $\begin{array}{c}-0,00550 \\
(0,135)\end{array}$ & $\begin{array}{c}0,0395 \\
(0,0894)\end{array}$ & $\begin{array}{c}0,156 \\
(0,116)\end{array}$ & $\begin{array}{c}-0,27 * * * \\
(0,0892)\end{array}$ & $\begin{array}{r}0,536 * * * \\
(0,119)\end{array}$ \\
\hline Patriotismo & $\begin{array}{c}-0,28 * * * \\
(0,0594)\end{array}$ & $\begin{array}{c}0,0383 \\
(0,0692)\end{array}$ & $\begin{array}{c}-0,32 * * * * \\
(0,0673)\end{array}$ & $\begin{array}{l}0,00247 \\
(0,0860)\end{array}$ & $\begin{array}{c}-0,27 * * * \\
(0,0695)\end{array}$ & $\begin{array}{c}0,0380 \\
(0,0799)\end{array}$ & $\begin{array}{c}-0,22 * * * \\
(0,0627)\end{array}$ & $\begin{array}{r}-0,0547 \\
(0,0761)\end{array}$ \\
\hline Seguridad & & $\begin{array}{c}-0,201 * * * \\
(0,0675)\end{array}$ & & $\begin{array}{l}0,0225 \\
(0,125)\end{array}$ & & $\begin{array}{l}-0,0695 \\
(0,0690)\end{array}$ & & $\begin{array}{c}-0,19 * * * \\
(0,0657)\end{array}$ \\
\hline Robo & & $\begin{array}{c}0,0116 \\
(0,0545)\end{array}$ & & $\begin{array}{c}-0,131 \\
(0,0813)\end{array}$ & & $\begin{array}{c}-0,161^{* *} \\
(0,0634)\end{array}$ & & $\begin{array}{c}0,0544 \\
(0,0552)\end{array}$ \\
\hline Zona Norte & $\begin{array}{c}-0,46 * * * \\
(0,113)\end{array}$ & $\begin{array}{c}0,609 * * * \\
(0,133)\end{array}$ & $\begin{array}{l}-0,181 \\
(0,138)\end{array}$ & $\begin{array}{c}0,303 \\
(0,186)\end{array}$ & $\begin{array}{l}0,0559 \\
(0,125)\end{array}$ & $\begin{array}{c}0,672 * * * \\
(0,153)\end{array}$ & $\begin{array}{c}-0,38 * * * \\
(0,108)\end{array}$ & $\begin{array}{c}0,495 * * * \\
(0,132)\end{array}$ \\
\hline Zona Centro & $\begin{array}{c}-0,228^{* *} \\
(0,0987)\end{array}$ & $\begin{array}{c}0,428 * * * \\
(0,125)\end{array}$ & $\begin{array}{c}0,00965 \\
(0,101)\end{array}$ & $\begin{array}{c}0,252 \\
(0,158)\end{array}$ & $\begin{array}{c}0,233^{* * *} \\
(0,101)\end{array}$ & $\begin{array}{c}0,778 * * * \\
(0,134)\end{array}$ & $\begin{array}{l}-0,189^{*} \\
(0,0984)\end{array}$ & $\begin{array}{c}0,340 * * * \\
(0,126)\end{array}$ \\
\hline Zona Sur & $\begin{array}{l}-0,168 \\
(0,118)\end{array}$ & $\begin{array}{c}0,805^{* * * *} \\
(0,134)\end{array}$ & $\begin{array}{c}0,352 * * * \\
(0,136)\end{array}$ & $\begin{array}{c}0,590^{* * * *} \\
(0,151)\end{array}$ & $\begin{array}{c}0,633 * * * \\
(0,112)\end{array}$ & $\begin{array}{c}1,058 * * * \\
(0,134)\end{array}$ & $\begin{array}{c}-0,195^{*} \\
(0,112)\end{array}$ & $\begin{array}{r}0,759^{* * *} \\
(0,128)\end{array}$ \\
\hline Cut11 & $\begin{array}{c}-1,62 * * * \\
(0,430)\end{array}$ & & $\begin{array}{c}-2,01^{* * *} \\
(0,589)\end{array}$ & & $\begin{array}{c}-2,49 * * * \\
(0,430)\end{array}$ & & $\begin{array}{c}-1,91^{* * *} \\
(0,409)\end{array}$ & \\
\hline Cut12 & $\begin{array}{c}-1,31 * * * * \\
(0,429)\end{array}$ & & $\begin{array}{c}-1,65^{* * *} \\
(0,575)\end{array}$ & & $\begin{array}{c}-2,13 * * * \\
(0,434)\end{array}$ & & $\begin{array}{c}-1,62 * * * \\
(0,409)\end{array}$ & \\
\hline Cut13 & $\begin{array}{c}-1,00 * * \\
(0,420)\end{array}$ & & $\begin{array}{c}-1,291 * * \\
(0,556)\end{array}$ & & $\begin{array}{c}-1,80^{* * * *} \\
(0,428)\end{array}$ & & $\begin{array}{c}-1,33 * * * \\
(0,402)\end{array}$ & \\
\hline Cut14 & $\begin{array}{c}-0,690^{*} \\
(0,411)\end{array}$ & & $\begin{array}{l}-0,910^{*} \\
(0,544)\end{array}$ & & $\begin{array}{c}-1,46 * * * \\
(0,425)\end{array}$ & & $\begin{array}{c}-1,02 * * * \\
(0,396)\end{array}$ & \\
\hline Cut15 & $\begin{array}{r}-0,320 \\
(0,404)\end{array}$ & & $\begin{array}{l}-0,459 \\
(0,533)\end{array}$ & & $\begin{array}{c}-1,057 * * \\
(0,425)\end{array}$ & & $\begin{array}{c}-0,676^{*} \\
(0,393)\end{array}$ & \\
\hline Cut16 & $\begin{array}{l}0,0999 \\
(0,398)\end{array}$ & & $\begin{array}{l}0,0502 \\
(0,517)\end{array}$ & & $\begin{array}{l}-0,606 \\
(0,427)\end{array}$ & & $\begin{array}{l}-0,273 \\
(0,390)\end{array}$ & \\
\hline Cut17 & $\begin{array}{c}0,590 \\
(0,395)\end{array}$ & & $\begin{array}{c}0,644 \\
(0,498)\end{array}$ & & $\begin{array}{c}-0,0896 \\
(0,430)\end{array}$ & & $\begin{array}{c}0,205 \\
(0,389)\end{array}$ & \\
\hline Cut18 & $\begin{array}{c}1,194 * * * \\
(0,394)\end{array}$ & & $\begin{array}{c}1,376^{* * *} \\
(0,473)\end{array}$ & & $\begin{array}{c}0,537 \\
(0,436)\end{array}$ & & $\begin{array}{c}0,799 * * \\
(0,391)\end{array}$ & \\
\hline Cut19 & $\begin{array}{c}1,595 * * * \\
(0,398)\end{array}$ & & $\begin{array}{c}1,862^{* * *} \\
(0,459)\end{array}$ & & $\begin{array}{c}0,947 * * \\
(0,443)\end{array}$ & & $\begin{array}{c}1,199 * * * \\
(0,396)\end{array}$ & \\
\hline Cut21 & $\begin{array}{c}0,342 \\
(0,577)\end{array}$ & & $\begin{array}{c}3,156 * * * \\
(0,675)\end{array}$ & & $\begin{array}{l}-0,114 \\
(0,603)\end{array}$ & & $\begin{array}{l}1,207 * * \\
(0,570)\end{array}$ & \\
\hline Athrho & $\begin{array}{c}-1,04 * * * \\
(0,214)\end{array}$ & & $\begin{array}{c}0,332 \\
(0,443)\end{array}$ & & $\begin{array}{c}0,958^{* * *} \\
(0,177)\end{array}$ & & $\begin{array}{c}-1,15^{* * * *} \\
(0,169)\end{array}$ & \\
\hline Log Pseudolikelihood & $-1947,24$ & & $-1812,50$ & & $-1887,98$ & & $-1934,1$ & \\
\hline Wald chi2 & 183,45 & & 96,49 & & 177,37 & & 287,39 & \\
\hline Obs. & 787 & & 787 & & 787 & & 787 & \\
\hline
\end{tabular}

Nota: Errores estándar robusto en paréntesis. $* * * \mathrm{p}<0,01, * * \mathrm{p}<0,05, * \mathrm{p}<0,1$. 
Para el caso del bienestar subjetivo medido como satisfacción con la vida, emergen algunas variables de control adicionales que resultan ser significativas. Se tiene que los individuos que tienen más hijos tienden a estar menos satisfechos con su vida, probablemente debido a la carga económica y de tiempo que esto implica. Asimismo, las personas con una ocupación laboral manifiestan niveles más altos de satisfacción con la vida. El trabajo no solo es visto como una fuente de generación de ingresos sino también como una forma de realización profesional y personal. Finalmente, los datos sugieren que los individuos que manifiestan profesar una religión más activamente reportan un menor nivel de satisfacción con la vida. Se argumenta que la religión puede ser vista como un vehículo para satisfacer una necesidad espiritual, y que su práctica puede hacer aflorar sentimientos de disconformidad y culpa que reducen la satisfacción con la vida.

Similarmente a los resultados encontrados para el caso del bienestar subjetivo medido como felicidad, mientras que la participación en organizaciones de autoayuda influye positivamente en el nivel de satisfacción con la vida, la participación en organizaciones recreativas reduce los niveles de satisfacción con la vida. Finalmente, no se encuentra una relación significativa entre participación en organizaciones económicas y satisfacción. Lo anterior ratifica lo encontrado para el caso de la felicidad, relevando la importancia de la existencia y promoción de instancias de participación de autoayuda en el bienestar inmaterial personal, y, por consiguiente, en su calidad de vida, medido a través su nivel de satisfacción. La discrepancia en los resultados entre organizaciones recreativas y de autoayuda puede responder a los distintos objetivos que persigue cada tipo de organización. Mientras la participación en organizaciones recreativas puede estar explicada por el interés propio de desarrollo personal en actividades como el deporte, artes y música, las personas que participan en organizaciones de autoayuda tales como organizaciones religiosas y de caridad, lo hacen de manera voluntaria y altruista con el objeto de brindar una ayuda desinteresada a los demás. De esta manera, en el primer grupo de organizaciones se podrían generar comportamientos competitivos que den origen a situaciones de estrés y frustración, los cuales afecten negativamente el bienestar de las personas. En contraste, las personas que participan en organizaciones de autoayuda podrían tener una sensación de complacencia o gratificación por contribuir a mejorar la situación de los demás.

Finalmente, la Tabla 5 presenta los resultados para el caso de la satisfacción con su situación económica. 


\section{TABLA 5}

\section{ESTIMACIONES PARA EL BIENESTAR SUBJETIVO MEDIDO COMO SATISFACCION CON SU SITUACION ECONOMICA}

\begin{tabular}{|c|c|c|c|c|c|c|c|c|}
\hline \multirow[t]{2}{*}{ Variables } & \multicolumn{2}{|c|}{ (1) } & \multicolumn{2}{|c|}{ (2) } & \multicolumn{2}{|c|}{ (3) } & \multicolumn{2}{|c|}{ (4) } \\
\hline & \multicolumn{8}{|c|}{ Participación en organizaciones } \\
\hline Todas & $\begin{array}{c}0,552 \\
(0,669)\end{array}$ & & & & & & & \\
\hline Económicas & & & $\begin{array}{c}1,381 * * * \\
(0,151)\end{array}$ & & & & & \\
\hline Recreativas & & & & & $\begin{array}{c}1,167^{* * * *} \\
(0,191)\end{array}$ & & & \\
\hline \multirow[t]{2}{*}{ Autoayuda } & & & & & & & $\begin{array}{c}1,179 * * * \\
(0,289)\end{array}$ & \\
\hline & \multicolumn{8}{|c|}{ Variables de control } \\
\hline Hombre & $\begin{array}{c}-0,203^{* *} \\
(0,0977)\end{array}$ & $\begin{array}{c}0,268 * * * \\
(0,104)\end{array}$ & $\begin{array}{c}-0,201 * * \\
(0,0804)\end{array}$ & $\begin{array}{c}0,176 \\
(0,111)\end{array}$ & $\begin{array}{c}-0,34 * * * \\
(0,0854)\end{array}$ & $\begin{array}{c}0,559 * * * \\
(0,110)\end{array}$ & $\begin{array}{l}-0,0618 \\
(0,0844)\end{array}$ & $\begin{array}{r}-0,189 * \\
(0,103)\end{array}$ \\
\hline Edad & $\begin{array}{c}0,0145 \\
(00169)\end{array}$ & $-0,0124$ & 0,00620 & 0,00601 & 0,0336 ** & $-0,07 * * *$ & 0,00242 & 0,0198 \\
\hline Edad2 & $-0,00017$ & 0,000116 & $-0,000117$ & $3,19 \mathrm{e}-05$ & $-0,0003^{*}$ & $0,0006 * *$ & $-7,33 \mathrm{e}-05$ & $-0,00013$ \\
\hline Educa & $\begin{array}{c}(0,00018) \\
0,0359 \\
(0,0374)\end{array}$ & $\begin{array}{c}(0,000214) \\
0,0595 \\
(0,0466)\end{array}$ & $\begin{array}{c}(0,0001 / 4) \\
0,0392 \\
(0,0359)\end{array}$ & $\begin{array}{c}(0,00024 l) \\
0,0103 \\
(0,0449)\end{array}$ & $\begin{array}{c}0,0328 \\
(0,0324)\end{array}$ & $\begin{array}{c}(0,0002) \\
0,0143 \\
(0,0424)\end{array}$ & $\begin{array}{c}(0,00018) \\
0,00584 \\
(0,0356)\end{array}$ & $\begin{array}{l}(0,0002) \\
0,0756^{*} \\
(0,0409)\end{array}$ \\
\hline Edad educa & $\begin{array}{l}0,00295 \\
(0,0169)\end{array}$ & $\begin{array}{c}0,0107 \\
(0,0216)\end{array}$ & $\begin{array}{l}-0,0121 \\
(0,0172)\end{array}$ & $\begin{array}{c}0,0428 * * \\
(0,0194)\end{array}$ & $\begin{array}{c}-0,00373 \\
(0,0140)\end{array}$ & $\begin{array}{c}0,0259 \\
(0,0173)\end{array}$ & $\begin{array}{l}0,00853 \\
(0,0154)\end{array}$ & $\begin{array}{r}-0,00549 \\
(0,0177)\end{array}$ \\
\hline Casado & $\begin{array}{c}0,0167 \\
(0,0828)\end{array}$ & $\begin{array}{l}-0,105 \\
(0,108)\end{array}$ & $\begin{array}{c}-0,000960 \\
(0,0793)\end{array}$ & $\begin{array}{c}-0,0305 \\
(0,116)\end{array}$ & $\begin{array}{r}-0,00547 \\
(0,0783)\end{array}$ & $\begin{array}{l}0,00622 \\
(0,112)\end{array}$ & $\begin{array}{l}0,00520 \\
(0,0810)\end{array}$ & $\begin{array}{l}0,0112 \\
(0,110)\end{array}$ \\
\hline $\mathrm{N}^{\circ}$ niños & $\begin{array}{l}-0,0125 \\
(0,0354)\end{array}$ & $\begin{array}{c}0,0606 \\
(0,0413)\end{array}$ & $\begin{array}{l}-0,0170 \\
(0,0309)\end{array}$ & $\begin{array}{c}0,0396 \\
(0,0439)\end{array}$ & $\begin{array}{l}-0,0129 \\
(0,0318)\end{array}$ & $\begin{array}{l}0,0682^{*} \\
(0,0415)\end{array}$ & $\begin{array}{l}-0,0297 \\
(0,0328)\end{array}$ & $\begin{array}{c}0,0608 \\
(0,0398)\end{array}$ \\
\hline Ocupado & $\begin{array}{l}0,0410 \\
(0,138)\end{array}$ & $\begin{array}{c}-0,437 * * * \\
(0,117)\end{array}$ & $\begin{array}{c}-0,124 \\
(0,0920)\end{array}$ & $\begin{array}{c}0,286^{* *} \\
(0,138)\end{array}$ & $\begin{array}{c}0,0148 \\
(0,0968)\end{array}$ & $\begin{array}{c}-0,0852 \\
(0,130)\end{array}$ & $\begin{array}{c}0,152 \\
(0,107)\end{array}$ & $\begin{array}{c}-0,41 * * * \\
(0,124)\end{array}$ \\
\hline Ahorro & $\begin{array}{c}0,413 * * * \\
(0,104)\end{array}$ & $\begin{array}{c}-0,264^{*} \\
(0,140)\end{array}$ & $\begin{array}{c}0,255^{* * *} \\
(0,0956)\end{array}$ & $\begin{array}{c}0,135 \\
(0,158)\end{array}$ & $\begin{array}{c}0,411^{* * *} \\
(0,0988)\end{array}$ & $\begin{array}{l}-0,208 \\
(0,144)\end{array}$ & $\begin{array}{c}0,407 * * * * \\
(0,104)\end{array}$ & $\begin{array}{l}-0,248^{*} \\
(0,138)\end{array}$ \\
\hline Ingresos & $\begin{array}{c}0,235 * * * \\
(0,0361)\end{array}$ & $\begin{array}{c}0,0391 \\
(0,0309)\end{array}$ & $\begin{array}{c}0,199 * * * \\
(0,0279)\end{array}$ & $\begin{array}{c}0,0678^{* * *} \\
(0,0337)\end{array}$ & $\begin{array}{c}0,205^{* * * *} \\
(0,0279)\end{array}$ & $\begin{array}{c}0,0501 \\
(0,0322)\end{array}$ & $\begin{array}{c}0,194 * * * * \\
(0,0380)\end{array}$ & $\begin{array}{c}0,0699 * * \\
(0,0322)\end{array}$ \\
\hline Religioso & $\begin{array}{c}0,104 \\
(0,156)\end{array}$ & $\begin{array}{c}0,478 * * * \\
(0,115)\end{array}$ & $\begin{array}{l}0,183^{* *} \\
(0,0882)\end{array}$ & $\begin{array}{l}0,0444 \\
(0,125)\end{array}$ & $\begin{array}{c}0,133 \\
(0,0891)\end{array}$ & $\begin{array}{c}0,148 \\
(0,118)\end{array}$ & $\begin{array}{c}-0,0649 \\
(0,117)\end{array}$ & $\begin{array}{c}0,583^{* * * *} \\
(0,128)\end{array}$ \\
\hline Patriotismo & $\begin{array}{c}-0,17 * * * \\
(0,0542)\end{array}$ & $\begin{array}{c}0,0462 \\
(0,0711)\end{array}$ & $\begin{array}{c}-0,159^{* * * *} \\
(0,0559)\end{array}$ & $\begin{array}{c}0,0674 \\
(0,0813)\end{array}$ & $\begin{array}{c}-0,16^{* * *} \\
(0,0532)\end{array}$ & $\begin{array}{c}0,0483 \\
(0,0742)\end{array}$ & $\begin{array}{c}-0,131^{* *} \\
(0,0578)\end{array}$ & $\begin{array}{l}-0,0242 \\
(0,0734)\end{array}$ \\
\hline Seguridad & & $\begin{array}{c}-0,178^{* *} \\
(0,0868)\end{array}$ & & $\begin{array}{l}-0,0958 \\
(0,0780)\end{array}$ & & $\begin{array}{c}-0,201^{* *} \\
(0,0710)\end{array}$ & & $\begin{array}{c}-0,178^{* *} \\
(0,0751)\end{array}$ \\
\hline Robo & & $\begin{array}{l}-0,0171 \\
(0,0839)\end{array}$ & & $\begin{array}{l}-0,110 \\
(0,0683)\end{array}$ & & $\begin{array}{l}-0,162^{* *} \\
(0,0648)\end{array}$ & & $\begin{array}{c}0,0189 \\
(0,0634)\end{array}$ \\
\hline Zona Norte & $\begin{array}{c}0,00346 \\
(0,185)\end{array}$ & $\begin{array}{c}0,635^{* * * *} \\
(0,146)\end{array}$ & $\begin{array}{c}-0,0128 \\
(0,106)\end{array}$ & $\begin{array}{c}0,352^{* *} \\
(0,156)\end{array}$ & $\begin{array}{l}-0,144 \\
(0,113)\end{array}$ & $\begin{array}{c}0,694 * * * \\
(0,145)\end{array}$ & $\begin{array}{l}-0,0546 \\
(0,117)\end{array}$ & $\begin{array}{c}0,408 * * * \\
(0,141)\end{array}$ \\
\hline Zona Centro & $\begin{array}{l}-0,107 \\
(0,148)\end{array}$ & $\begin{array}{c}0,456^{* * * *} \\
(0,131)\end{array}$ & $\begin{array}{l}-0,0857 \\
(0,0979)\end{array}$ & $\begin{array}{l}0,237^{*} \\
(0,139)\end{array}$ & $\begin{array}{c}-0,264 * * \\
(0,109)\end{array}$ & $\begin{array}{c}0,662 * * * \\
(0,133)\end{array}$ & $\begin{array}{l}-0,158 \\
(0,107)\end{array}$ & $\begin{array}{c}0,330 * * \\
(0,133)\end{array}$ \\
\hline Zona Sur & $\begin{array}{l}-0,200 \\
(0,227)\end{array}$ & $\begin{array}{c}0,860^{* * * *} \\
(0,144)\end{array}$ & $\begin{array}{c}-0,225^{* *} \\
(0,108)\end{array}$ & $\begin{array}{c}0,521^{* * *} \\
(0,143)\end{array}$ & $\begin{array}{c}-0,43^{* * *} \\
(0,126)\end{array}$ & $\begin{array}{c}1,034 * * * \\
(0,141)\end{array}$ & $\begin{array}{c}-0,36^{* * * *} \\
(0,131)\end{array}$ & $\begin{array}{c}0,743 * * * \\
(0,132)\end{array}$ \\
\hline Cut11 & $\begin{array}{r}-0,306 \\
(0,481)\end{array}$ & & $\begin{array}{c}-0,958^{* *} \\
(0,435)\end{array}$ & & $\begin{array}{l}0,0350 \\
(0,425)\end{array}$ & & $\begin{array}{l}-0,434 \\
(0,434)\end{array}$ & \\
\hline
\end{tabular}




\begin{tabular}{|c|c|c|c|c|}
\hline Variables & & & & $(4$ \\
\hline Cut12 & $-0,0429$ & $-0,733^{*}$ & 0,270 & $-0,203$ \\
\hline & $(0,464)$ & $(0,431)$ & $(0,415)$ & $(0,429)$ \\
\hline Cut13 & 0,511 & $-0,256$ & $0,768^{*}$ & 0,283 \\
\hline & $(0,446)$ & $(0,434)$ & $(0,407)$ & $(0,432)$ \\
\hline Cut14 & 1,090 ** & 0,249 & $1,293 * * *$ & $0,794^{*}$ \\
\hline & $(0,438)$ & $(0,440)$ & $(0,406)$ & $(0,445)$ \\
\hline Cut15 & $1,788^{* * *}$ & $0,866^{*}$ & $1,924 * * *$ & $1,412 * * *$ \\
\hline & $(0,436)$ & $(0,449)$ & $(0,411)$ & $(0,469)$ \\
\hline Cut16 & $\begin{array}{c}2,187 * * * \\
(0,436)\end{array}$ & $\begin{array}{c}1,223 * * * \\
(0,454)\end{array}$ & $\begin{array}{c}2,286^{* * * *} \\
(0,413)\end{array}$ & $\begin{array}{c}1,766 * * * \\
(0,484)\end{array}$ \\
\hline Cut17 & $\begin{array}{c}2,713 * * * * \\
(0.439)\end{array}$ & $\begin{array}{c}1,700 * * * \\
(0.460)\end{array}$ & $\begin{array}{c}2,763 * * * \\
(0.417)\end{array}$ & $\begin{array}{c}2,235 * * * \\
(0.505)\end{array}$ \\
\hline Cut18 & $\begin{array}{c}3,365 * * * \\
(0,450)\end{array}$ & $\begin{array}{c}2,295^{* * *} \\
(0,469)\end{array}$ & $\begin{array}{c}3,353 * * * \\
(0,427)\end{array}$ & $\begin{array}{c}2,819^{* * *} \\
(0,536)\end{array}$ \\
\hline Cut19 & $3,830^{* * *}$ & $2,724 * * *$ & $3,777^{* * * *}$ & $3,232 * * *$ \\
\hline & $(0,459)$ & $(0,470)$ & $(0,433)$ & $(0,554)$ \\
\hline Cut21 & $\begin{array}{c}0,195 \\
(0,669)\end{array}$ & $\begin{array}{c}2,765 * * * * \\
(0,629)\end{array}$ & $\begin{array}{l}-0,635 \\
(0,617)\end{array}$ & $\begin{array}{c}1,297 * * \\
(0.609)\end{array}$ \\
\hline Athrho & $\begin{array}{l}-0,304 \\
(0,447)\end{array}$ & $\begin{array}{c}-0,973 * * * * \\
(0,163)\end{array}$ & $\begin{array}{c}-0,79 * * * \\
(0,195)\end{array}$ & $\begin{array}{c}-0.81 * * * * \\
(0.309)\end{array}$ \\
\hline Log Pseudolikelihood & $-2021,45$ & $-1879,61$ & $-1962,2$ & -2012.8 \\
\hline Wald chi2 & 230,31 & 365,57 & 348,71 & 304.99 \\
\hline Obs. & 794 & 794 & 794 & 794 \\
\hline
\end{tabular}

Nota: Errores estándar robusto en paréntesis. *** $\mathrm{p}<0,01$, ** $\mathrm{p}<0,05, * \mathrm{p}<0,1$.

Finalmente, para el caso de la satisfacción con la situación económica, destacan como variables de control relevantes el sexo del individuo y los ingresos. Para el primer caso se tiene que los hombres tienden a manifestar un menor nivel de satisfacción económica que las mujeres. Lo anterior podría responder a diferencias en aspectos aspiracionales entre los hombres y mujeres, donde los hombres emergen como más ambiciosos y menos conformistas económicamente que las mujeres. Una relación positiva y significativa entre los ingresos y la satisfacción económica es esperada debido a la asociación estrecha existente entre ambas medidas.

Para el caso de la participación en organizaciones, a diferencia de los resultados anteriores, las estimaciones sugieren un efecto positivo y significativo en la satisfacción con su situación económica tanto para las organizaciones recreativas y de autoayuda como las organizaciones económicas. Este resultado podría estar mostrando los beneficios económicos que ofrece la participación en organizaciones de toda índole, por medio de las redes de personas que se concretan en estas instancias. Lo anterior no necesariamente se encuentra relacionada con potenciales vínculos afectivos, sino más bien con relaciones interpersonales y de reciprocidad que favorecen el acceso a la información y la reducción de costos de transacción, para de esta manera mejorar el bienestar material de las personas. 


\section{CONCLUSIONES}

Los Gobiernos han evolucionado a una mirada más integral del concepto de bienestar, incorporando elementos de carácter subjetivo en su medición. De esta manera, esfuerzos relacionados con la consecución de objetivos propiamente materiales están siendo redireccionados a acciones que sean capaces de realzar el bienestar global de las personas.

Uno de los factores clave para el desarrollo de toda sociedad es su capital social. En particular, la participación como medida de capital social no solo entrega a sus miembros mayores oportunidades para el acceso a recursos, promoviendo comportamientos cooperativos en pos de la consecución de beneficios económicos, sino también fomenta una vida social más activa con resultados positivos en los niveles de satisfacción y/o felicidad de las personas.

Este trabajo investiga esta última asociación a través de la estimación de modelos de respuesta ordenada bivariada para el bienestar subjetivo y participación social para distintas medidas de bienestar y categorías de organizaciones.

Los resultados indican una asociación positiva y significativa entre la participación en organizaciones sociales de distinta índole y la medida de bienestar subjetiva relacionada con la satisfacción y con la situación económica. Lo anterior podría ser consecuencia de las mayores oportunidades que ofrecen las organizaciones para concretar redes de colaboración, y de esta manera, mejorar el acceso a la información y reducir los costos de transacción. Sin embargo, cuando se explora el efecto de la participación social en la satisfacción con la vida y felicidad, medidas mucho más subjetivas de bienestar, los resultados muestran un efecto positivo solo para las organizaciones de autoayuda. El efecto no es conclusivo para las organizaciones económicas. Lo anterior es consistente con la visión de que la participación en organizaciones económicas responde mayormente a un interés en mejorar condiciones laborales y productivas, lo cual no necesariamente se traduce en una mejora en el bienestar desde el punto de vista de la satisfacción con la vida y felicidad. Los resultados también muestran una asociación negativa de la participación en organizaciones recreativas con las medidas de satisfacción con la vida y felicidad. De esta manera, mientras las personas participan en organizaciones de autoayuda con la motivación de brindar apoyo desinteresado a los demás por medio de acciones de caridad, religiosas o ayuda humanitaria, los individuos desarrollan actividades de recreación por placer y desafío personal. Así, puede ser más probable que las organizaciones de auto ayuda canalicen de mejor manera instancias sociales de interacción y generosidad que pueden dar como resultado la concreción de relaciones afectivas y experiencias positivas que aumenten los niveles de satisfacción con la vida y felicidad. Por otra parte, en las organizaciones recreativas pueden dominar sentimientos de competencia y estrés que podrían tener una incidencia negativa en el bienestar. Lo anterior no contradice la evidencia encontrada en Salazar y Jaime (2009), cuando la participación en organizaciones económicas tiene una incidencia positiva en la satisfacción con la situación económica de las personas. Este trabajo 
contribuye a generalizar el rol clave que juegan las organizaciones sociales, no solo en realzar el bienestar material, sino también los niveles de satisfacción y felicidad de la población, especialmente aquellas orientadas a promover acciones de caridad, religiosas y de ayuda humanitaria.

\section{REFERENCIAS}

AKER, J. (2007). Social Networks and Household Welfare in Tanzania: Working Together to Get Out of Poverty (SSRN Scholarly Paper No ID 995941). Rochester, NY: Social Science Research Network.

BASMANN, R. (1960). "On finite sample distributions of generalized classical linear identifiability test statistics". Journal of the American Statistical Association, 55 (292), pp. 650-59.

BHARADWAJ, L. y E. WILKENING (1977). "The prediction of perceived well-being”. Social Indicators Research, 4 (1), pp. 4-421.

CALVO, E. y P. BEYTIA (2011). “¿Cómo medir la felicidad?”. Claves para Políticas Públicas, 4, pp. 1-9.

COLEMAN, J. (1988). "Social Capital in the Creation of Human Capital". American Journal of Sociology, 94, pp. 95-120.

DIENER, E. (1994). "Assessing subjective well-being: Progress and opportunities". Social Indicators Research, 31 (2), pp. 103-157.

DIENER, E. y M. SELIGMAN (2002). "Very Happy People”. Psychological Science, 13 (1), pp. 81-84.

DIENER, E.; R. LUCAS; U. SCHIMMACK y J. HELLIWELL (2009). "Well-being for public policy". Oxford: Oxford University Press.

DOLAN, P.; T. PEASGOOD y M. WHITE (2008). "Do we really know what makes us happy? A review of the economic literature on the factors associated with subjective well-being". Journal of Economic Psychology, 29 (1), pp. 94:122.

DURSTON, J. (1999). "Construyendo capital social comunitario". Revista de la CEPAL 69, pp. 103-118.

FLEESON, W.; A. MALANOS y N. ACHILLE (2002). "An intra-individual process approach to the relationship between extraversion and positive affect: Is acting extraverted as 'good' as being extraverted?". Journal of Personality and Social Psychology, 83 (6), pp. 1409-1422.

GREENE, W. (1999). "Econometric Analysis". Macmillan, New York.

HELLIWELL, J. (2001). "Social capital, the economy and well-being". In: The review of economic performance and social progress 2001: The longest decade: Canada in the 1990s.

HERREROS, F. (2002). “ ¿Son las relaciones sociales una fuente de recursos? Una definición del capital social", Papers 67, pp. 129-148.

HUDSON, J. (2006). "Institutional trust and subjective well-being across the EU". Kyklos, 59 (1), pp. 43-62.

IDH (2012). "Bienestar subjetivo: el desafío de repensar el desarrolloen Chile". Recuperado 26 de Abril de 2016, a partir de http://desarrollohumano.cl/idh/informes/2012-bienestar-subjetivo-el-desafio-de-repensar-el-desarrollo/

KLIKSBERG, B. (1999). "Capital social y cultura, claves esenciales del desarrollo". Comisión Económica para América Latina y el Caribe. Retrieved April 26, 2016, from http://repositorio.cepal.org/ handle/11362/12190

KNACK, S. y P. KEEFER (1997). "Does Social Capital Have an Economic Payoff? A Cross-Country Investigation". The Quarterly Journal of Economics, 112 (4), pp. 1251:1288.

PNUD (Programa de las Naciones Unidas para el Desarrollo (PNUD) (2000). "Asociatividad y capital social". Desarrollo Humano en Chile, pp. 107-172.

PONCE, M.; R. ROSAS y M. LORCA (2014). "Social capital, social participation and life satisfaction among Chilean older adults". Revista de Saúde Pública, 48 (5), pp. 739-749.

PORTELA, M. y I. NEIRA (2012). "Capital social y bienestar subjetivo. Un análisis para España considerando sus regiones". Investigaciones Regionales, 23, pp. 5-27.

PUNTSCHER, S.; C. HAUSER; J. WALDE y G. TAPPEINER (2014). "The impact of social capital on subjective well-being: A regional perspective”. Journal of Happiness Studies, 16 (5), pp. 1231-1246. 
PUTNAM, R. (1995). "Bowling alone: American's declining social capital”. Journal of Democracy, 6 (1), pp. 65-78.

SALAZAR, C. y M. JAIME (2009). "Participación en organizaciones sociales en Chile ¿Una alternativa para mejorar el bienestar económicos de los hogares en Chile?”. Estudios de Economía, 36 (2), pp. 191-215.

VEENHOVEN, R. (1994). "El estudio de la satisfacción con la vida". Intervención Psicosocial, 3, pp. 87-116.

WORLD VALUE SURVEY (2012). <www.worldvaluessurvey.org> 